Check for updates

Cite this: RSC Adv., 2017, 7, 53689

\title{
Synthesis of artificial chaperones in a novel type of Pickering emulsion for glycoprotein $\dagger$
}

\begin{abstract}
Tao Zhao, (D) Wenjun Wen, Junping Wang (D) and Shuo Wang
Novel particles of poly(DVB-co-PBA) were synthesized by a one-step solvothermal route instead of distillation precipitation polymerization (DPP), which was simple to operate. The poly(DVB-co-PBA) showed excellent versatility in the preparation of functional materials. On the one hand, it could be applied to the preparation of a sugar fluorescent sensor due to the features of molecular recognition of cis-diol-containing compounds and fluorescence; on the other hand, it showed flexibility in the preparation of a Pickering emulsion. Further, the core-shell microspheres named W-type and W-II type were synthesized in emulsions of water-in-toluene and water-in-chloroform with stabilizers of poly(DVB-co-PBA), respectively. The core-shell microspheres named O-type were synthesized in the emulsion of oil-in-water with stabilizers poly(DVB-co-PBA) aL-Cys. The W-type polymer microspheres were used as thermally stable materials for the glycoprotein of horseradish peroxidase (HRP) to study the change of conformation and catalytic activity of HRP under heat stress through methods of UV-vis spectrophotometry, circular dichroism spectroscopy, ANS probe, chromogenic substrates, FITC fluorescence labelling, TEM and enzyme catalysis reaction. The results showed the W-type polymer microspheres with a core of hydrophilic/hydrophobic moieties reaching $2 / 1$ could effectively maintain the natural conformation of HRP and longer catalytic activity under heat stress.
\end{abstract}

Received 19th October 2017

Accepted 10th November 2017

DOI: $10.1039 / c 7 r a 11558 j$

rsc.li/rsc-advances protein protection and refolding. For instance, Masahiko and coworkers ${ }^{15}$ have synthesized polymer nanoparticles (NPs) capable of facilitating resolubilization and refolding of an aggregated protein of positively charged lysozyme. The NPs could be prepared by copolymerizing optimized combinations and populations of functional monomers. Polymer micelles could be used as a substitute for surfactants to promote protein refolding. Huang and co-workers ${ }^{16}$ have synthesized the mixed-shell polymeric micelles (MSPMs) which were used as a suppressor of Alzheimer's disease. The balance of hydrophilic/hydrophobic moieties on the surface of MSPMs was important for their enhanced therapeutic effect. The amphiphilicity of cyclodextrin and polysaccharides was very important for the refolding of the protein, especially the balance between the hydrophilic and hydrophobic properties played a key role in inhibiting the aggregation of protein monomers. ${ }^{17}$ Pickering emulsion as a two-phase or multi-phase system, which has a great advantage in the preparation of the amphiphilic materials. However, there were few reports on the preparation of artificial chaperones in Pickering emulsions.

Pickering emulsion is an emulsion that stabilized by solid particles in place of surfactants. ${ }^{18}$ In the past studies, inorganic particles and organic particles of a single component were used as stabilizers for Pickering emulsions, such as silica, ${ }^{19}$ LAPONITE ${ }^{\circ}$ clay, $^{20}$ zinc oxide ${ }^{21}$ titania $^{22}$ and polystyrene latex particles. $^{23}$ There were limited reports on the organic hybrid particles as stabilizers. Multi-component organic particles endow the Pickering emulsion more features for the preparation of
Key Laboratory of Food Nutrition and Safety, Ministry of Education, Tianjin University of Science \& Technology, 29 The Thirteenth Road, Tianjing Economy and Technology Development Area, Tianjin, 300457, PR China. E-mail: wangjp@tust.edu.cn; wangshuo@nankai.edu.cn; Fax: +86 22 60601332; +86 22 83534450; Tel: +86 22 6060145; +862283534450

$\dagger$ Electronic supplementary information (ESI) available. See DOI: 10.1039/c7ra11558j 
versatile hybrid materials, especially in the preparation of amphiphilic materials for protein protection.

In this study, three types of Pickering emulsion were first prepared based on the stabilizers of poly(DVB-co-PBA) microspheres which were synthesized by the method of distillation precipitation polymerization (DPP). ${ }^{24}$ To demonstrate the flexibility of the multi-component organic particles microspheres of poly(DVB-co-PBA) in the preparation of hybrid materials, three hybrid polymer microspheres of core-shell structure were prepared in three types of emulsions by Pickering emulsion polymerization, respectively. The amphiphilic polymer microspheres of similar pine cone structure were used as thermal stabilizers to study the change of conformation and catalytic activity of horseradish peroxidase (HRP) under heat stress.

\section{Experimental}

\section{Materials}

Divinylbenzene (DVB, 80\%, technical grade, mixture of isomers) was purchased from Sigma-Aldrich (Germany), 2,2'-azodiisobutyronitrile (AIBN), acrylamide (AM), $N, N, N^{\prime}, N^{\prime}$-tetramethylethylenediamine (TEMED), benzoylperoxide, $N, N^{\prime}$-methylenebis(acrylamide) (MBAA), 4-vinylphenylboronic acid, $\mathrm{N}$-isopropylacrylamide (NIPAAm), fluorescein isothiocyanate (FITC), guaiacol and 8-anilino-1-naphthalenesulfonic acid ammonium salt $\left(\right.$ ANS-NH $\left.{ }_{4}\right)$ were obtained from Sinopharm Chemical Reagent Co., Ltd. (Shanghai, China), horseradish peroxidase (HRP), L-cysteine and ammonium persulfate (APS) were purchased from Sigma-Aldrich (USA).

\section{Synthesis of poly(DVB-co-PBA) particles by DPP}

The poly(DVB-co-PBA) particles were first fabricated by the DPP technique. In detail, the monomers, 4 -vinyl phenylboronic acid $(1.48 \mathrm{~g}, 10 \mathrm{mmol})$ and divinylbenzene $(1.30 \mathrm{~g}, 10 \mathrm{mmol})$, and initiator AIBN (60 mg, $0.37 \mathrm{mmol}$ ) were dissolved in $100 \mathrm{~mL}$ of acetonitrile in around-bottom flask. The mixture was purged with pure $\mathrm{N}_{2}$ for $10 \mathrm{~min}$. And then the Dean-Stark receiver was connected to the flask. After that the flash heated to $70{ }^{\circ} \mathrm{C}$ until the reaction solution appears cloudy in an oil bath. The polymerized reaction was proceeded under reflux with the gradually increase of the oil bath to $110{ }^{\circ} \mathrm{C}$. The reaction was stopped when half of the reaction solvent was distilled through the Dean-Stark receiver within $2 \mathrm{~h}$. The flask was removing from the oil bath. The resultant product was filtered with a sand core funnel of G5, and then the products were washed with methanol for three times to remove the residual oligomers and monomers, then vacuum drying.

\section{Synthesis of poly(DVB-co-PBA) particles by one-step solvothermal}

The poly(DVB-co-PBA) particles were fabricated by the solvothermal method. In detail, the monomers, 4-vinyl phenylboronic acid (735 mg, $5 \mathrm{mmol})$ and DVB $(710 \mu \mathrm{L}, 5 \mathrm{mmol})$, and initiator AIBN $(30 \mathrm{mg})$ were dissolved in $80 \mathrm{~mL}$ of acetonitrile in reaction kettle. The mixture was purged with pure $\mathrm{N}_{2}$ for $10 \mathrm{~min}$. The polymerized reaction was carried out at $110{ }^{\circ} \mathrm{C}$ for
$8 \mathrm{~h}$. The resultant product was filtered with a sand core funnel of G5, and then the product was washed with methanol for three times to remove the residual oligomers and monomers, then vacuum drying.

\section{Synthesis of poly(DVB-co-PBA)@L-Cys particles}

The poly(DVB-co-PBA) particles $(100 \mathrm{mg})$, L-cysteine $(50 \mathrm{mg})$ and AIBN (5 mg), were dispersed in the ethanol-water $(2: 3, \mathrm{v} / \mathrm{v})$ mixture in a round-bottomed flask. After purged with pure $\mathrm{N}_{2}$ for $10 \mathrm{~min}$, the mixture was heated to $70{ }^{\circ} \mathrm{C}$ for $24 \mathrm{~h}$ in an oil bath. When the flask was removed from the oil bath, the reaction was stopped. The product was washed repeatedly with ethanol-water mixture and the pure poly(DVB-co-PBA)@L-Cys particles were collected by centrifugation.

\section{Synthesis of W-type polymer microspheres}

The poly(DVB-co-PBA) particles (200 mg) were dissolved in $15 \mathrm{~mL}$ toluene as an oil phase, and then ultrasound for 10 minutes. AM (150 mg), MBAA (10 mg) and NIPAAm (50 mg) were dissolved in $5 \mathrm{~mL}$ DDW as a water phase. After mixing the two phases, shaking vigorously for $5 \mathrm{~min}$ by hand. The initiator of APS $(10 \mathrm{mg})$ and TEMED $(20 \mu \mathrm{L})$ were added to emulsion, the polymerization reaction was carried out at $30{ }^{\circ} \mathrm{C}$ for $24 \mathrm{~h}$. After polymerization, the solvent was removed by decantation. The resultant product was filtered with a sand core funnel of G5, and then the products were washed with methanol for three times to remove the residual oligomers and monomers, then vacuum drying.

\section{Synthesis of W-II type polymer microspheres}

The poly(DVB-co-PBA) particles $(50 \mathrm{mg})$ were dissolved in $15 \mathrm{~mL}$ chloroform as an oil phase, and then ultrasound for 10 minutes. AM (150 mg), MBAA (10 mg) and NIPAAm (50 mg) were dissolved in $10 \mathrm{~mL}$ DDW as a water phase. After mixing the two phases, shaking vigorously for $5 \mathrm{~min}$ by hand. The initiator of APS $(10 \mathrm{mg})$ and TEMED $(20 \mu \mathrm{L})$ were added to emulsion, the polymerization reaction was carried out at $30{ }^{\circ} \mathrm{C}$ for $24 \mathrm{~h}$. The purification process was consistent with the above.

\section{Synthesis of O-type polymer microspheres}

The poly(DVB-co-PBA) @L-Cys $(20 \mathrm{mg})$ was dissolved in $3 \mathrm{~mL}$ distilled water (DDW) as a water phase, the initiator of benzoylperoxide $(4.0 \mathrm{mg}$ ) was dissolved in $1.5 \mathrm{~mL}$ divinylbenzene as an oil phase. After mixing the two phases, shaking vigorously for 5 min by hand, polymerization was conducted for 14 hours at room temperature. After polymerization, the resultant product was filtered with a sand core funnel of G5, and then the products were washed with methanol for three times to remove the residual oligomers and monomers, then vacuum drying.

\section{Result and discussion}

\section{Synthesis and characterization of poly(DVB-co-PBA)}

The poly(DVB-co-PBA) microspheres were first synthesized by the method of distillation precipitation polymerization (DPP). ${ }^{18}$ To simplify the synthesis process, one-step solvothermal was 
A
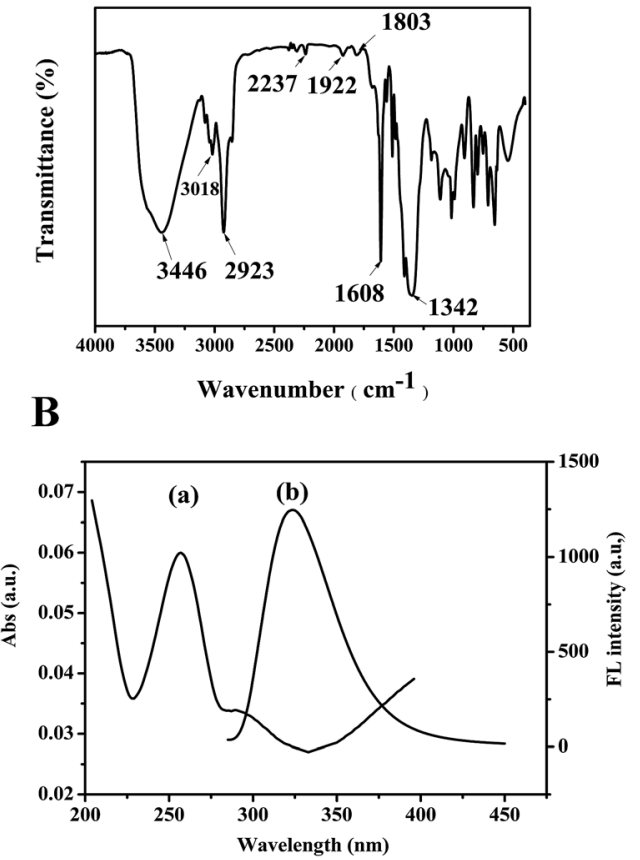

Fig. 1 FT-IR spectrum (A), the UV-visible absorption spectra and emission spectrum (B) of poly(DVB-co-PBA) microsphere.

used to prepare the poly(DVB-co-PBA) microspheres instead of the method of DPP. The one-step solvothermal which used the begin time as clear criteria for the initial of the polymerization reaction was easier to operate compared to the method of DPP. The poly(DVB-co-PBA) microspheres with good morphology (Fig. S1 $\dagger$ ) were fabricated by one-step solvothermal.

Fig. 1(A) was the FT-IR spectrum of the polymer, the peaks of 3226 and $1342 \mathrm{~cm}^{-1}$ were the stretching of the $-\mathrm{OH}$ of the boronic acid group, the peak of 3018 and $1608 \mathrm{~cm}^{-1}$ were the $\mathrm{C}-\mathrm{H}$ bond and $\mathrm{C}=\mathrm{C}$ of the benzene ring, respectively. The feature of $2923 \mathrm{~cm}^{-1}$ was $\mathrm{C}-\mathrm{H}$ of the methylene of straight chain $\left(-\mathrm{CH}_{2}-\right)$, the feature of $2237 \mathrm{~cm}^{-1}$ was B-O bond, the feature of 1803 and $1922 \mathrm{~cm}^{-1}$ were $\mathrm{C}-\mathrm{H}$ and $\mathrm{C}-\mathrm{C}$ vibrations $(\mathrm{R}-\mathrm{CH}=\mathrm{CH})$ of straight chain, respectively. The results indicated that poly (DVB-co-PBA) has been successfully synthesized. Fig. 1(B) show the UV-visible absorption spectra and emission spectrum of poly(DVB-co-PBA). The polymer microspheres have the maximum UV absorption peak at $258 \mathrm{~nm}$. The fluorescence emission spectra of the polymer microspheres were obtained by using $258 \mathrm{~nm}$ as the excitation wavelength. The maximum emission wavelength was found at $323.5 \mathrm{~nm}$.

The results in Fig. $2 \mathrm{a}$ and $\mathrm{b}$ indicated that the morphology of the polymer microspheres was structured. The size of poly(DVB-coPBA) microspheres ranged from 1 to $2 \mu \mathrm{m}$. The effective diameter was $1385.3 \mathrm{~nm}$ (Fig. S2 $\dagger$ ). The characterization aspects of carbohydrate affinity and TGA were detailed in ESI (Fig. S3 and S4 $\dagger$ ).

\section{The poly(DVB-co-PBA) as stabilizer of Pickering emulsion}

The synthetic poly(DVB-co-PBA) microspheres as stabilizer had some new features compared to the inorganic particles and

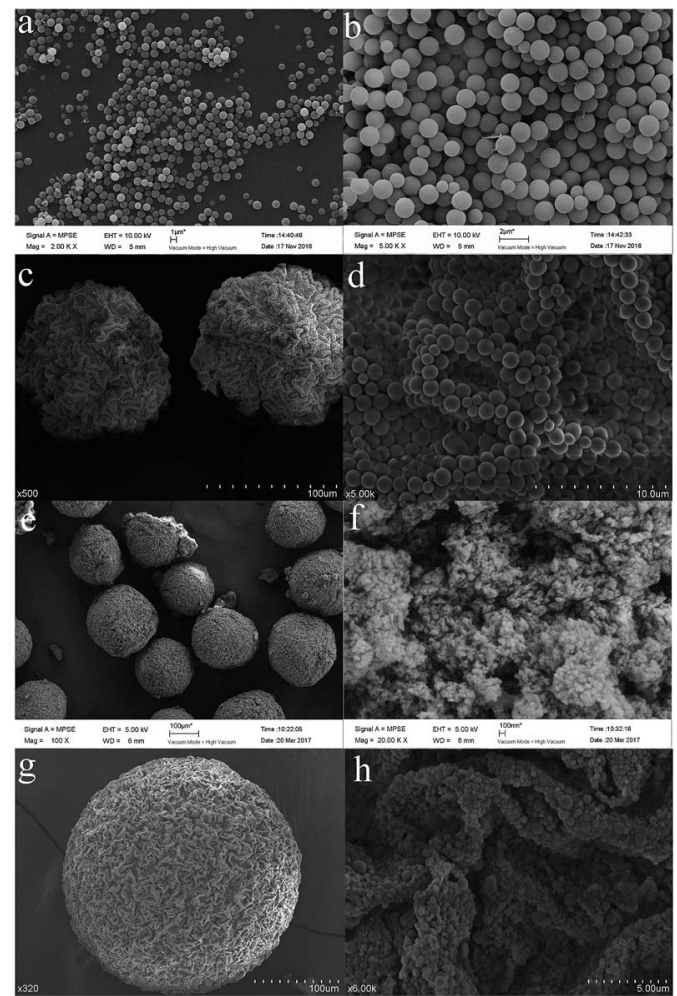

Fig. 2 SEM of poly(DVB-co-PBA) microspheres (a) 2000x and (b) $5000 \times$; the W-type of amphiphilic polymer microspheres (c) $500 \times$ and the surface of the $W$-type of amphiphilic polymer microspheres (d) $5000 \times$; the W-II type of amphiphilic polymer microspheres (e) $100 \times$ and the surface of the $\mathrm{W}$-II type of amphiphilic polymer $r$ microspheres (f) $20 \mathrm{k} \times$; the O-type polymer microspheres (g) $320 \times$ and the surface of the $\mathrm{W}$-II type of amphiphilic polymer $r$ microspheres (h) $6 \mathrm{k} \times$.

organic particles of a single component. On one hand, due to the hydrophilicity and carbohydrate affinity of the boronic acid groups, the poly(DVB-co-PBA) microspheres were more hydrophilic than the polystyrene microspheres. The contact angle of water droplet was $52.7^{\circ}$ (Fig. S5 $\dagger$ ). The poly(DVB-co-PBA) microspheres were easier to form Pickering emulsions without the addition of other chemical agents in conventional organic reagents, which could form two kinds of water-in-oil Pickering emulsions of water-in-toluene and water-in-chloroform. The phenylboronic acid as carbohydrate affinity agent, which endow the poly(DVB-co-PBA) microsphere the feature of molecular recognition of cis-diol-containing compounds. ${ }^{25,26}$ On the other hand, the synthetic poly(DVB-co-PBA) microspheres were susceptible to chemical modification. We used the poly(DVB-coPBA) @L-Cys microspheres as the stabilizers for the preparation of a type of oil-in-water Pickering emulsion.

\section{Preparation of the core-shell microspheres in the water-in- toluene emulsion}

In the system of water-in-toluene, acrylamide (AM), $N$-isopropylacrylamide (NIPAAm) and $N, N^{\prime}$-methylenebis(acrylamide) were dissolved in distilled water (DDW) as the aqueous phase, the poly(DVB-co-PBA) microspheres were added to toluene as the oil phase, the water-in-toluene Pickering emulsion was 
prepared by mixing the two phases, and then the initiator was added to Pickering emulsion for polymerization. Finally, we obtained a type of amphiphilic particle with core-shell structure after polymerization. The surface was composed of hydrophobic particles of poly(DVB-co-PBA) microspheres and the core was composed of a hydrophilic hydrogel (Fig. 2c and d). This type of the core-shell microsphere was referred simply as $\mathrm{W}$-type.

\section{Preparation of the core-shell microspheres in the water-in- chloroform emulsion}

In the system of water-in-chloroform, we tried to use the corrosion characteristic of chloroform to reduce the size of the surface microspheres and improve its porosity. A novel type of amphiphilic core-shell structure microsphere which had a nanoscale surface was fabricated by Pickering emulsion polymerization (Fig. 2e and f). This type of the core-shell microspheres was referred simply as W-II type.

\section{Preparation of the core-shell microspheres in the oil-in-water emulsion}

For further study, L-cysteine was grafted on the surface of the poly(DVB-co-PBA) microspheres by "click chemistry". ${ }^{27}$ We used the poly(DVB-co-PBA)@L-Cys as stabilizers to prepare an oil-inwater Pickering emulsion. We used the oil-in-water Pickering emulsion to prepare a novel core-shell strong hydrophobic polymer microsphere that had hydrophobic core of poly(DVB) and was enveloped by the shell of poly(DVB-co-PBA)@L-Cys particles by Pickering emulsion polymerization (Fig. $2 g$ and $h$ ). This type of the core-shell microspheres was referred simply as O-type.

The synthetic poly(DVB-co-PBA) microspheres showed versatile for the preparation of Pickering emulsion. Three types of microspheres of core-shell structure with different morphology and properties were prepared by Pickering emulsion polymerization. The W-type microspheres with well-arranged structure undergo a conformational switching by water absorbing swelling (Fig. S7 $\dagger$ ). The W-II type which had a nanoscale surface could be stable in the water for a long time without damage, the reason may be the chloroform corrosiveness enhancing the binding between the core and the shell (Fig. S8†). The O-type microspheres that had many "gully" structures in the surface may be used as a good adsorbent.

What is more, the core in the microspheres of core-shell structure could be prepared by copolymerizing optimized combinations and populations of functional monomers, which endow the hybrid materials more features. The synthesis process of the three types of core-shell microspheres were detailed in ESI (Fig. S6 and S9†).

\section{The protective function of $\mathrm{W}$-type polymer microspheres for natural structure of HRP}

We used the W-type microsphere as thermal stabilizers to study the change of conformation and catalytic activity of HRP under heat stress.

The balance of hydrophilic/hydrophobic moieties is important for maintaining protein conformation. Acrylamide as a hydrophilic functional monomer and $N$-isopropylacrylamide as a hydrophobic functional monomer, the balance of hydrophilic/hydrophobic moieties of W-type materials was controlled by changing the proportions of acrylamide (AM) and $\mathrm{N}$-isopropylacrylamide (NIPAAm) in the core of the W-type microspheres. Four types of the W-type microspheres of the different proportions of AM and NIPAAm (the molar ratio of AM/NIPAAm: all AM, $4: 1,2: 1$, all NIPAAm). The synthesis process is detailed in ESI (Table S1 $\dagger$ ). The materials of all AM and $4: 1$ were used as the hydrophilic control groups, the materials of $2: 1$ were used as the neutral control groups, the materials of all NIPAAm and the O-type microspheres were used as the hydrophobic control groups.

\section{The analysis of UV-vis spectrophotometer}

The groups of adding W-type microspheres could maintain good UV-vis peak of horseradish peroxidase (HRP) compared with the blank group after the adsorption process of a long time (Fig. S11 $\dagger$ ). In consideration of this phenomenon, we assumed that $\mathrm{W}$-type microspheres could maintain the conformation of the protein. To evaluate the properties of the W-type microspheres for maintaining protein conformations stability, HRP was used as a test protein and the W-type microspheres of different proportions of AM and NIPAMM were used as thermal stabilizers through the methods of circular dichroism spectrum (CD), ANS probe, chromogenic substrates, and FITC fluorescence label under heating conditions. Despite a lot of elegant works on the thermal stability materials for protein, there were few reports about the microspheres of core-shell structure for maintaining thermal stability of glycoprotein.

\section{The analysis of circular dichroism (CD)}

To evaluate the capability of the $\mathrm{W}$-type polymer microspheres for maintaining the natural conformation of protein, horseradish peroxidase (HRP) solution $\left(1 \mathrm{mg} \mathrm{mL}^{-1}, \mathrm{pH}=7.4\right.$, PBS buffer) with different types of materials were heated at $60{ }^{\circ} \mathrm{C}$ for $30 \mathrm{~min}$, then the solution was filtered with $0.22 \mu \mathrm{m}$ water filters and determined by using CD spectrophotometer.

The contents of the secondary structure of the horseradish peroxidase in the group of $2: 1$ was similar to the group of untreated HRP (Table 1). For the strong hydrophilic groups (all AM, $4: 1$ ) and the strong hydrophobic groups (all NIPAAm), the capability of maintaining protein conformation under heat stress was weak. Especially when the hydrophobicity was too strong (O-type, poly(DVB-co-PBA)), the hydrophobic force could cause irreversible denaturation.

The results indicated that the W-type microspheres could maintain the secondary structure of glycoprotein in the heating conditions only when the hydrophilic/hydrophobic moieties reached a certain equilibrium. There were two reasons that may lead to this result. On one hand, the protein monomers through the hydrophobic interaction to form the natural structure, the materials that lack of hydrophobic force were not conducive for protein refolding. On the other hand, the hydrophilic environment provided a living environment for the protein, too strong hydrophobic effect would denature the protein and accelerate irreversible aggregation. 
Table 1 Secondary structure content of HRP with addition of different materials under heat stress

\begin{tabular}{lcccc}
\hline Result & Helix & Strand & Turns & Unordered \\
\hline Untreated HRP & 0.349 & 0.112 & 0.233 & 0.319 \\
Denatured HRP & 0.209 & 0.247 & 0.195 & 0.382 \\
All AM & 0.209 & 0.242 & 0.191 & 0.370 \\
$4: 1$ & 0.130 & 0.252 & 0.180 & 0.400 \\
$2: 1$ & 0.347 & 0.119 & 0.247 & 0.330 \\
All NIPAAm & 0.805 & 0.006 & 0.066 & 0.160 \\
O-type & 0.800 & 0.006 & 0.071 & 0.169 \\
Poly(DVB-co-PBA) & 0.793 & 0.007 & 0.075 & 0.162
\end{tabular}

\section{The analysis of ANS probe}

To further verify the capability of the W-type microspheres for maintaining the conformation of HRP under heating conditions. ANS probe which could be combined into the exposed hydrophobic region of the protein in water was utilized to characterize part of unfolded intermediates of protein.

The ANS probe has a maximum excitation wavelength at $350 \mathrm{~nm}$ and a maximum emission wavelength at $514 \mathrm{~nm}$ when present alone. However, the maximum emission wavelength becomes $460 \mathrm{~nm}$ when combined into the hydrophobic region of the protein, corresponding to a significant enhancement of the fluorescence intensity. The result in Fig. 3 showed that only the group of (c) and the unheated group of (HRP) did not occur a blue shift. The results indicated that the material in group of (c) had the ability to stable the conformation of protein, which could reduce the hydrophobic area of HRP under heat stress denaturation.

Table 2 showed the changes of the maximum fluorescence emission peak of ANS. The denatured HRP was much like the groups of ALL AM. The result indicated materials that composed of all hydrophilic monomers are less capable of maintaining the native structure of the glycoprotein. Meanwhile, the degree of blue shift of the strong hydrophobic groups (all NIPAAm, poly(DVB-co-PBA)) were the maximum. The results

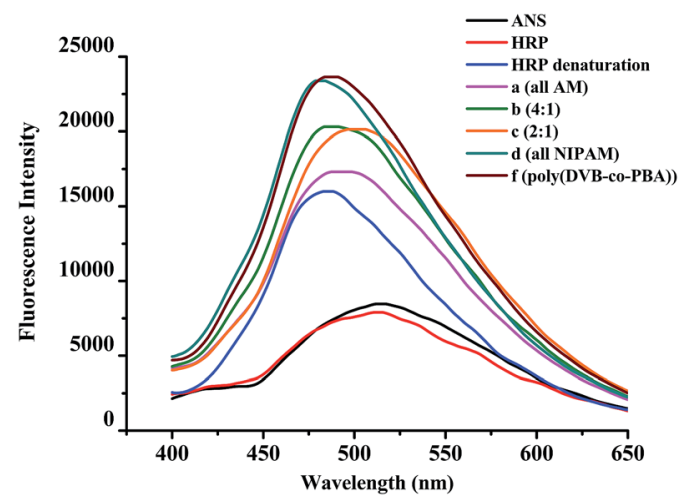

Fig. 3 Effect of stabilizers on fluorescence of ANS binding to HRP incubated at $60{ }^{\circ} \mathrm{C}$ for $0.5 \mathrm{~h}$ in the presence and absence of stabilizers. HRP: unheated group, HRP denaturation: heat-denatured in absence of stabilizers, $(a-f)$ heat-denatured HRP with addition of different materials.
Table 2 Fluorescence Intensity of ANS binding to HRP with addition of different materials under heat stress

\begin{tabular}{llc}
\hline Result & Wavelength $(\mathrm{nm})$ & Fluorescence intensity (a.u.) \\
\hline ANS & 511 & 8458.9 \\
Untreated HRP & 511 & 7897.5 \\
Denatured HRP & 483 & 15996.6 \\
All AM & 499 & 17315.6 \\
$4: 1$ & 491 & 20308.4 \\
$2: 1$ & 506 & 20135.9 \\
All NIPAAm & 479 & 23385.1 \\
Poly(DVB-co-PBA) & 479 & 23647.0
\end{tabular}

indicated too much hydrophobicity would accelerate the protein denaturation process these results were also consistent with the CD spectrophotometer.

\section{The analysis of chromogenic substrates}

To verify that the W-type microspheres could not only sustain the secondary structure of the HRP but also maintain a certain activity of the HRP under heat stress. The HRP-DAB chromogenic reagent was used to evaluate the capability of the material for maintaining the activity of HRP protein under heating conditions.

The color effect of group (c) was significantly superior to other control groups (Fig. 4). The results indicated that heatdenatured HRP in group (c) maintained a certain activity. Due to the activity of HRP is closely related to its tertiary structure, the W-type polymer microspheres of group (c) may have the capability of maintaining a certain tertiary structure of HRP under heating conditions.

\section{The analysis of FITC-HRP}

For further study, the FITC labeled HRP were used to study the superficial mechanism of thermal protection for glycoprotein. The solution of FITC-HRP were added into the flat bottles with different types of materials and then heated at $60{ }^{\circ} \mathrm{C}$ for half an hour. After heating, the mixture was dropped to a slide and observed with a confocal fluorescence microscope.

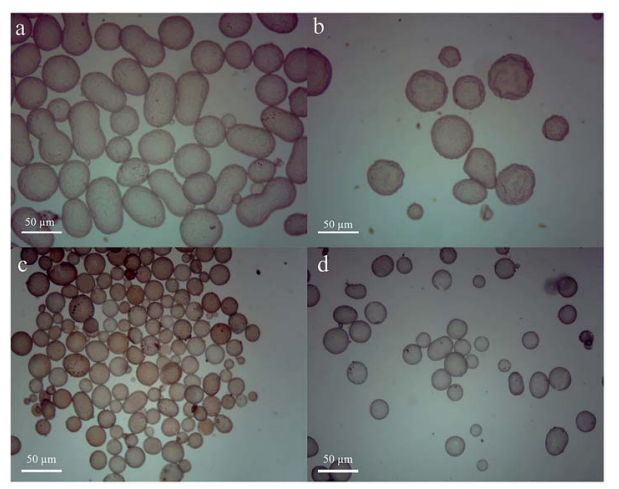

Fig. 4 Optical micrographs of the W-type polymer microspheres with the heat-denatured HRP and DAB-HRP chromogenic reagent $(10 \times)$, (a-d) all AM, $4: 1,2: 1$, all NIPAAm (the molar ratio of AM/NIPAAm of the W-type microspheres). 


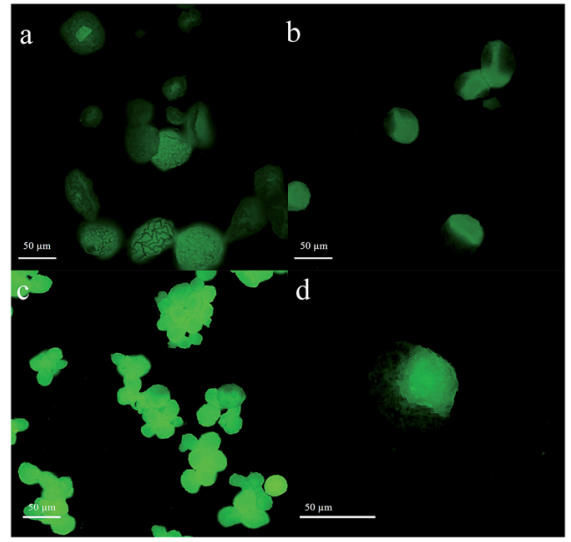

Fig. 5 Confocal fluorescence microscope images of the W-type polymer microspheres with the heat-denatured FITC-HRP, $(\mathrm{a}-\mathrm{c})$ all AM, 4:1, 2:1 (the molar ratio of AM/NIPAM of the W-type microspheres, $10 \times)$, (d) the broken material of $4: 1(40 \times)$.

The fluorescence intensity of FITC-HRP were the highest in the group of (c) (Fig. 5c), the results indicated that the $\mathrm{W}$-type polymer microspheres of group (c) had the best capability of maintaining a certain structure of HRP under heating conditions, which were consistent with the other experimental results. The fluorescence intensity of the core of the W-type polymer microspheres was significantly higher than the surface (Fig. 5d). The results indicated that the FITC-HRP entered the internal hydrogel through the channel of the surface during the heating process. The hydrogel core of W-type polymer microspheres was indispensable for thermal protection.

Based on the verified results, we proposed a hypothesis that the W-type polymer microspheres as thermal stabilizers were like a surfactant. The W-type polymer microspheres could form a structure like reverse micelles directly in the aqueous phase. The internal hydrogel as a "water pool" could provide a survival environment for protein, the denatured proteins that were trapped to the internal hydrogel without aggregations. ${ }^{28}$ The hydrophobic surface of poly(DVB-co-PBA) was comparable to a protective cover, which could effectively reduce the thermal impact on the protein.

\section{The analysis of TEM}

The state of heat-denatured HRP in presence of different types of materials were studied by TEM, the process was detailed in ESI. $\dagger$

The result in Fig. $\mathrm{S} 12 \dagger$ showed that the group of unheated HRP (Fig. S12(a) $\dagger$ ) did not occur aggregation. But for the heating denaturation HRP without adding materials, many black spots occurred (Fig. S12(b) †). The reason may be that heating denaturation led to aggregation of protein monomers, which resulted in an irreversible deposition. For the groups of $\mathrm{c}-\mathrm{f}$, which were added with different types of W-type polymer microspheres, the particle size of HRP was small and no serious aggregation occurred. This result indicated that the materials of W-type had the capability of maintaining a certain structure of HRP under heating conditions. For the hydrophobic groups of $f$ and $g$, it was found that a small amount of protein was present
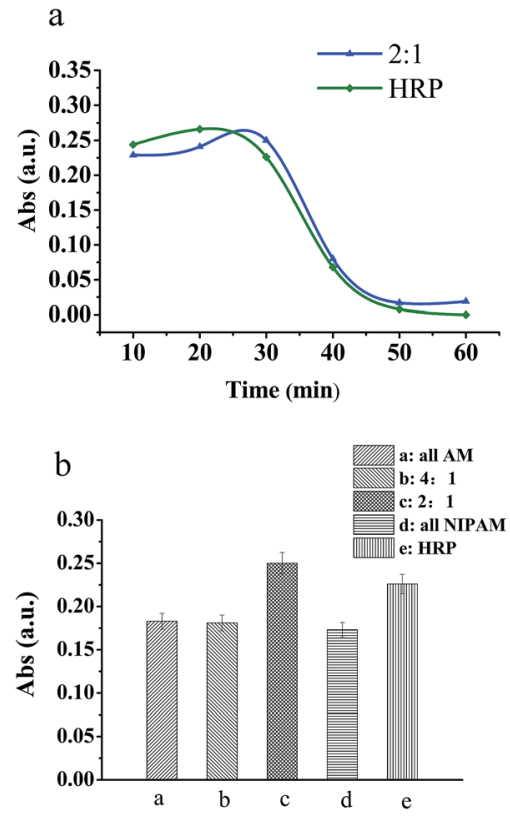

Fig. 6 Changes of catalytic activity of HRP, (a): changes of catalytic activity of HRP under continuous heating in presence and absence of thermal stabilizers, (b): the catalytic activity of HRP after heated at $60{ }^{\circ} \mathrm{C}$ for $30 \mathrm{~min}$.

in the aggregation state. It indicated that strong hydrophobic materials were not conducive to the conformational stability of the protein during the heating process.

\section{The protection for catalytic activity of HRP under heat stress}

In order to investigate the catalysis of HRP in presence of the Wtype polymer microspheres which were used as thermal stabilizers under heat stress. The catalyzing reaction of $\mathrm{H}_{2} \mathrm{O}_{2}$ oxidizing guaiacol as a test reaction. The assay procedure was detailed in ESI. $\dagger$

The results in Fig. 6(a) showed the catalytic activity of the group which is in absence of thermal stabilizers (Fig. 6 (HRP)) entered a linear descend section at 25 minutes of continuous heating, but for the group which is in the presence of thermal stabilizers (Fig. $6(2: 1)$ ) was 30 minutes. The results indicated that W-type material could improve the thermostable temperature of the HRP, and which could maintain longer catalytic activity at high temperatures. Fig. 6(b) showed the catalytic activity of HRP after heated at $60{ }^{\circ} \mathrm{C}$ for half an hour. The group c $(2: 1)$ had the best ability to stabilize catalytic activity under heating conditions. This result indicated that the W-type microspheres could maintain the catalytic activity of HRP in the heating conditions only when the hydrophilic/hydrophobic moieties reached a certain equilibrium, which was consistent with other experimental results.

\section{Conclusions}

In summary, one-step solvothermal instead of the method of DPP successfully to synthesize the poly(DVB-co-PBA) which was a kind of new multi-function organic particle being used as the 
stabilizer of Pickering emulsion. The synthetic poly(DVB-coPBA) microspheres of multi-component exhibited versatile compared with the inorganic particles and organic particles of a single component in the preparation of hybrid materials by Pickering emulsion polymerization. The synthetic poly(DVB-coPBA) with some new features of regular morphology, fluorescence, carbohydrate affinity and easier to form different types of Pickering emulsions. Three kinds of microspheres of core-shell structure were prepared in three types of emulsions, respectively. The $\mathrm{W}$-type of amphiphilic polymer microsphere with a core of the hydrophilic/hydrophobic moieties reached to $2 / 1$ could suppresses HRP thermal aggregation with high efficiency and longer catalytic activity of HRP under heat stress. Further studies on the deep mechanism of thermal protection for glycoprotein are now in progress.

\section{Conflicts of interest}

There are no conflicts to declare.

\section{Acknowledgements}

We gratefully appreciate the financial support by the National Key Research and Development Program of China (no. 2016YFD0401202). Special Fund for Grain-Scientific Research in the Public Interest (201513006-04). The National Key Technology R\&D Program (2015BAD17B03).

\section{Notes and references}

1 K. Nishinari, Y. Fang, S. Guo and G. O. Phillips, Food Hydrocolloids, 2014, 39, 301-318.

2 K. N. Ryan and E. A. Foegeding, Food Hydrocolloids, 2015, 43, 265-274.

3 H. B. Wijayanti, N. Bansal and H. C. Deeth, Compr. Rev. Food Sci. Food Saf., 2014, 13, 1235-1251.

4 O. E. Mäkinen, E. Zannini, P. Koehler and E. K. Arendt, Food Chem., 2016, 196, 17-24.

5 N. T. Wright and J. D. Humphrey, Annu. Rev. Biomed. Eng., 2002, 4, 109-128.

6 B. Song, Q. Sun, H. Li, B. Ge, J. S. Pan, A. T. S. Wee and F. Huang, Angew. Chem., Int. Ed., 2014, 126, 6476-6481.
7 G. Stirnemann, S. G. Kang, R. Zhou and B. J. Berne, Proc. Natl. Acad. Sci. U. S. A., 2014, 111, 3413-3418.

8 D. B. Wetlaufer and Y. Xie, Protein Sci., 1995, 4, 1535-1543.

9 H. Shimizu, K. Fujimoto and H. Kawaguchi, Biotechnol. Prog., 2000, 16, 248-253.

10 K. Akiyoshi, A. Yoshihiro Sasaki and J. Sunamoto, Bioconjugate Chem., 1999, 10, 321.

11 X. Liu, Y. Liu, Z. Zhang, F. Huang, Q. Tao, R. Ma, Y. An and L. Shi, Chem.-Eur. J., 2013, 19, 7437-7442.

12 Y. Sasaki and K. Akiyoshi, Curr. Pharm. Biotechnol., 2010, 11, 300-305.

13 H. Takahashi, S. Sawada and K. Akiyoshi, ACS Nano, 2011, 5, 337-345.

14 M. Sakono, T. Maruyama, N. Kamiya and M. Goto, Biochem. Eng. J., 2004, 19, 217-220.

15 M. Nakamoto, T. Nonaka, K. J. Shea, Y. Miura and Y. Hoshino, J. Am. Chem. Soc., 2016, 138, 4282.

16 F. Huang, J. Wang, A. Qu, L. Shen, J. Liu, J. Liu, Z. Zhang, Y. An and L. Shi, Angew. Chem., Int. Ed., 2014, 53, 8985-8990.

17 S. Takeda, H. Takahashi, S. I. Sawada, Y. Sasaki and K. Akiyoshi, RSC Adv., 2013, 3, 25716-25718.

18 R. Aveyard, B. P. Binks and J. H. Clint, Adv. Colloid Interface Sci., 2003, 100, 503-546.

19 J. Frelichowska, M. A. Bolzinger and Y. Chevalier, Colloids Surf., A, 2009, 343, 70-74.

20 N. P. Ashby and B. P. Binks, Phys. Chem. Chem. Phys., 2000, 2, 5640-5646.

21 Y. He, Powder Technol., 2004, 147, 59-63.

22 X. Song, Y. Zhao, H. Wang and Q. Du, Langmuir, 2009, 25, 4443-4449.

23 B. P. Binks and S. O. Lumsdon, Langmuir, 2001, 17, 45404547.

24 F. Bai, X. Yang and W. Huang, Macromolecules, 2004, 37, 3641-3649.

25 D. Li, Y. Chen and Z. Liu, Chem. Soc. Rev., 2015, 44, 80978123.

26 T. Zhao, J. Wang, J. He, Q. Deng and S. Wang, Biosens. Bioelectron., 2017, 91, 756-761.

27 L. L. Guo, Q. X. Li, X. Tang, K. G. Neoh and E. T. Kang, Macromolecules, 2010, 43, 5797-5803.

28 N. Morimoto, T. Endo, Y. Iwasaki and K. Akiyoshi, Biomacromolecules, 2005, 6, 1829-1834. 